\title{
Blimp-1 Mediates Tracheal Lumen Maturation in Drosophila melanogaster
}

\author{
Arzu Öztürk-Çolak, ${ }^{*, t, 1}$ Camille Stephan-Otto Attolini, ${ }^{\dagger}$ Jordi Casanova, ${ }^{*,+, 2}$ and Sofia J. Araújo*,t,t,2 \\ *Institut de Biologia Molecular de Barcelona (IBMB-CSIC), Parc Cientific de Barcelona, C. Baldiri Reixac 10, 08028 Barcelona, Spain, \\ †'Institut de Recerca Biomedica de Barcelona (IRB Barcelona), The Barcelona Institute of Science and Technology, C. Baldiri Reixac \\ 10, 08028 Barcelona, Spain, and `Department of Genetics, Microbiology and Statistics, Faculty of Biology, University of Barcelona, \\ 08028 Barcelona, Spain and Institute of Biomedicine University of Barcelona (IBUB) \\ ORCID IDs: 0000-0001-8045-320X (C.S.-O.A.); 0000-0002-4749-8913 (S.J.A.)
}

\begin{abstract}
The specification of tissue identity during embryonic development requires precise spatio-temporal coordination of gene expression. Many transcription factors required for the development of organs have been identified and their expression patterns are known; however, the mechanisms through which they coordinate gene expression in time remain poorly understood. Here, we show that hormoneinduced transcription factor Blimp-1 participates in the temporal coordination of tubulogenesis in Drosophila melanogaster by regulating the expression of many genes involved in tube maturation. In particular, we demonstrate that Blimp-1 regulates the expression of genes involved in chitin deposition and F-actin organization. We show that Blimp-1 is involved in the temporal control of lumen maturation by regulating the beginning of chitin deposition. We also report that Blimp-1 represses a variety of genes involved in tracheal maturation. Finally, we reveal that the kinase Btk29A serves as a link between Blimp-1 transcriptional repression and apical extracellular matrix organization.
\end{abstract}

KEYWORDS Blimp-1; Drosophila; trachea; chitin; Btk29A; aECM

PECIALIZED cellular functions and cell lineage fates are usually regulated by only a few key instructive transcription factors required to activate or repress specific patterns of gene expression. During the development of multicellular organisms, these events must be precisely timed. As a result, organogenesis requires an accurate spatio-temporal regulation of gene expression over extended periods. While many transcription factors required for the development of organs have been identified and their expression pinpointed spatially, the mechanisms through which they coordinate downstream gene expression in time remain poorly understood. Here, we addressed part of this question during the development of the Drosophila melanogaster tracheal system-a model used to study epithelial organ development. The tracheal system of

Copyright @ 2018 by the Genetics Society of America

doi: https://doi.org/10.1534/genetics.118.301444

Manuscript received July 3, 2018; accepted for publication August 1, 2018; published Early Online June 8, 2018.

Supplemental material available at Figshare: https://doi.org/10.25386/genetics.6736427.

${ }^{1}$ Present address: Department of Neuroscience, The Farber Institute for Neurosciences, Kimmel Cancer Center, Thomas Jefferson University, Philadelphia, PA 19107

${ }^{2}$ Corresponding authors: Institut de Biologia Molecular de Barcelona (IBMB-CSIC), The Barcelona Institute of Science and Technology, School of Biology, 08028 Barcelona, Spain. E-mail: sofiajaraujo@ub.edu; and jcrbmc@ibmb.csic.es
D. melanogaster is formed by a network of epithelial tubes that requires tight temporal regulation of gene expression. Tracheal tube maturation involves the timely and spatially regulated deposition of a chitinous apical extracellular matrix (aECM), a process governed by downstream effectors of the midembryonic ecdysone hormone pulse (Chavoshi et al. 2010). One of these ecdysone response genes is the $D$. melanogaster B-lymphocyte inducing maturation protein-1 (Blimp-1) (Ng et al. 2006; Chavoshi et al. 2010). Blimp-1 is the homolog of human Prdm1 (positive regulatory domain containing 1) (Huang 1994). Blimp-1/PRDM1 is a zinc finger transcriptional repressor that belongs to the Prdm gene family, and it was originally identified as a silencer of $\beta$-interferon gene expression (Keller and Maniatis 1991). Prdm family members contain a conserved N-terminal domain, known as a positive regulatory (PR) domain. This domain has been associated with the SET methyltransferase domain, which is important for the regulation of chromatin-mediated gene expression (Hohenauer and Moore 2012). In addition, Prdm family proteins contain multiple zinc fingers that mediate sequence-specific DNA binding and protein-protein interactions (Turner et al. 1994). Prdm family members modulate key cellular processes, including cell fate, and the aberrant 
function of some members may lead to malignant transformation (Fog et al. 2012). During embryonic development, Blimp-1 controls a plethora of cell-fate decisions in many organisms (Bikoff et al. 2009; Hohenauer and Moore 2012). In D. melanogaster, Blimp-1 serves as an ecdysone-inducible gene that regulates ftz-f1 in pupal stages (Agawa et al. 2007). By acting as a transcriptional repressor, Blimp-1 prevents the premature expression of $f t z-f 1$, thereby influencing the temporal regulation of events that are crucial for insect development. The expression level and stability of Blimp- 1 is critical for the precise timing of pupariation (Akagi et al. 2016).

Blimp-1 exerts a function in tracheal system morphogenesis during embryonic development (Ng et al. 2006; ÖztürkÇolak et al. 2016). However, the question remains as to how this transcription factor regulates tube maturation events downstream of the hormone ecdysone. Here, we studied the role of Blimp-1 in the transcriptional regulation of the regulation of tracheal tube maturation in D. melanogaster. We found that Blimp-1 is a transcriptional repressor of many genes involved in tracheal development and that its levels are critical for the precise timing of luminal maturation and the final stages of tubulogenesis in the embryo. Our results indicate that Blimp-1, working downstream of ecdysone, acts as a link of hormone action during tube maturation in organogenesis.

\section{Materials and Methods}

\section{D. melanogaster strains and genetics}

All D. melanogaster strains were raised at $25^{\circ}$ under standard conditions. Mutant chromosomes were balanced over LacZ or GFP-labeled balancer chromosomes. Overexpression and rescue experiments were carried out either with btl-GAL4 (kindly provided by M. Affolter) or AbdB-GAL4 (kindly provided by E. Sánchez-Herrero) drivers at 25 or $29^{\circ} \cdot y^{1} w^{118}$ (used as wild type), Blimp-1 ${ }^{K G 09531}$, and UAS-srcGFP are described in FlyBase; UAS-Blimp-1 (Öztürk-Çolak et al. 2016); Btk29A $\mathrm{A}^{\mathrm{k} 00206}$ and UAS-Btk29A (kindly provided by M. Strigini).

\section{Embryo staging and synchronization}

Embryos were staged following Campos-Ortega and Hartenstein (1985). To study temporal chitin deposition, cages were set at $25^{\circ}$ for $2 \mathrm{hr}$, and embryos were then allowed to develop for $18,20,22$ or $24 \mathrm{hr}$ at $18^{\circ}$ in order to obtain early and late stages 12 , and early and late 13 , respectively. Blimp-1 mutant embryos were compared to Blimp-1 heterozygotes after staining with CBP and 2A12. Heterozygote embryos were differentiated from homozygous mutant embryos by the presence or absence of a $\beta$-Gal-expressing balancer.

\section{Synthesis of pri/tal RNA probes for in situ hybridization}

The pri/tal RNA probes were synthesized using a PCR-based technique. The pri gene region [524 bp, covering all coding open reading frames (ORFs) of the gene] was defined, and the forward (5'TAATACGACTCACTATAGGTTTTTGGTCAATAC ACGGCA3') and reverse (5'AATTAACCCTCACTAAAGGAGTT
TGTGGATAAGGCACGG3') primers were designed accordingly so that the PCR product carried the two RNA promoters T3 and T7. The gene region of interest, flanked by the T3 and T7 sequences, was amplified from previously isolated genomic DNA via PCR under standard PCR conditions. The newly synthesized RNA was then purified by precipitation, resuspended in hybridization buffer, and stored at $-20^{\circ}$.

\section{Immunohistochemistry, image acquisition, and processing}

Standard protocols for immunostaining were applied. The following antibodies were used: rat anti-DE-cad (DCAD2, DSHB); rabbit anti-GFP (Molecular Probes); anti-Gasp mAb2A12 (DSHB); guinea pig anti-Blimp-1(S. Roy); antiexpansion and anti-rebuf (from M. Llimargas); anti-knk (from A. Uv) anti Btk29A (from M. Strigini); anti aPKC (Santa Cruz Biotechnology); and chicken anti- $\beta$-gal (Cappel). Biotinylated or Cy3-, Cy2-and Cy5-conjugated secondary antibodies (Jackson ImmunoResearch) were used at 1:300. Chitin was visualized with Fluostain (Sigma) at $1 \mu \mathrm{g} / \mathrm{ml}$ or CBP (Chitin Binding Probe, our own, made according to NEB protocols). Confocal images of fixed embryos were obtained either with a Leica TCS-SPE, a Leica TCS-SP2, or a Leica TCS-SP5 system. Images were processed using Fiji and assembled using Photoshop. 3D cell shape reconstructions were done using Imaris software. Quantifications of apical cell surface areas were done using Fiji (Schindelin 2012).

\section{Fluorescent in situ hybridization}

Freshly fixed embryos were washed and kept at $56^{\circ}$ in hybridization buffer for $3 \mathrm{hr}$ for prehybridization. In the last 10 min of prehybridization, probes (1:100 in hybridization Buffer) were prepared for hybridization. The probes were hybridized with the embryos at $56^{\circ}$ overnight. The next day the embryos were washed and incubated in POD-conjugated anti-DIG (in PBT) for $1 \mathrm{hr}$. The fluorescent signal was developed by the addition of Cy3 Amplification Reagent (1:100) diluted in TSA amplification diluent and incubation at room temperature in the dark for $10 \mathrm{~min}$. Finally, the embryos were either mounted in Fluoromount medium or subjected to antibody staining.

\section{In silico analysis of Blimp-1 binding sites}

The Blimp-1 position weight matrix was taken from the reported binding sequences in Kuo and Calame (2004). We extracted sequences 2000 bp upstream and downstream from all annotated isoforms in the $D$. melanogaster genome using the ENSEMBL (Cunningham et al. 2015) and biomaRt (Smedley et al. 2015) databases. All computations were performed within the R statistical framework (http://www.Rproject.org). The Matscan software (Blanco et al. 2006) was used to find putative binding sites for the Blimp-1 position weight matrix in the aforementioned regions. For each binding site, we computed the mean conservation score of the corresponding positions following the $D$. melanogaster related species' conservation track of the UCSC browser 
(Fujita et al. 2011). For genes with multiple transcription start sites (TSSs), nonredundant binding candidates for all TSSs were reported. Each gene was assigned with the maximum Matscan score of the corresponding binding sites after filtering by conservation score. Fisher tests were performed to find enrichment of tracheal genes among genes with predicted binding sites. All isoforms were included in the computation in order to consider multiple bindings for the same gene as this may increase the probability of binding and therefore tighter regulation.

\section{Data availability}

Fly strains are available upon request. Supplemental files contain the complete lists of possible genes/isoforms regulated by Blimp-1 and full information on the possible binding sites (Supplemental Material, Tables S1-S3), a supplemental file with all information on the files, and Figures S1 and S2. Supplemental material available at Figshare: https://doi. org/10.25386/genetics.6736427.

\section{Results}

\section{Blimp-1 modulates tracheal tube size and apical ECM formation}

Blimp-1, an ecdysone response gene (Beckstead et al. 2005; Chavoshi et al. 2010) (Figure S1), encodes the D. melanogaster homolog of the transcriptional factor B-lymphocyteinducing maturation protein gene, whose mutants have been reported to have misshapen trachea with severe defects in taenidia (Ng et al. 2006; Öztürk-Çolak et al. 2016). Detailed expression analysis of Blimp-1 protein in tracheal cells showed that expression is not detectable until embryonic stage 12 and is then observed until stage 15 (Figure 1, A-D). At this stage, Blimp-1 protein levels started decreasing, first in dorsal trunk (DT) cells, and by stage 16 the protein was no longer detectable in any tracheal cell. In addition to its tracheal expression, Blimp-1 was also detected in the epidermal, midgut, and hindgut cells throughout these stages (Figure 1, A-D). In Blimp-1 mutant stage 16 embryos, we detected lower levels of chitin, inflated tracheal tubes, and disorganized taenidial ridges (Öztürk-Çolak et al. 2016) (Figure 1, E-H).

The tube shape of Blimp-1 mutant embryos was altered, DTs showing a smaller diameter at the fusion points than in the rest of the tubes (Figure 1H). To better study whether this phenotype was due to constrictions at fusion points and/or dilations along the entire length of the tubes, we measured the tube diameter at fusion points of tracheal metameres $\operatorname{Tr} 7-\operatorname{Tr} 8$ and $\operatorname{Tr} 8-\operatorname{Tr} 9$ and the largest tube diameter at the Tr8 metamere between fusion points. Blimp-1 mutant embryos had a significantly ( $P$-value: $4.4 \mathrm{E}-07$, by Student's $t$-test, $n=10$ ) larger tube diameter between fusion points than wild-type $(w t)$ DTs $(n=15)$, while, at the fusion points, the tube diameter was similar to the wt (Figure 1I). These results suggest that Blimp-1 is required to maintain the luminal structures from overexpanding in between fusion points. Since tube expansion is related to the apical cell surface, we next examined cell shape and apical area in the trachea of Blimp-1 mutant embryos. We labeled the apical cell junctions in Blimp-1 mutant embryos and found that the apical cell shape differed to that of $w t$ cells (Figure 1, J-N). In Blimp-1 mutants, the longest cell axis appeared to be perpendicular to the tube axis, while in the wt it was parallel (Figure 1, J-M). Most of the Blimp-1 mutant cells had a reduced apical cell surface area when compared to wt cells, thereby suggesting a uniform organization throughout the tube (except for the fusion cells, which already had a distinct apical cell shape that did not seem to be affected by the loss of function of Blimp-1) (Figure 1K). To have a better idea of cell organization in Blimp-1 mutant trachea, we traced tracheal cell surfaces and compared them to those of the $w t$. We observed altered cell shapes in the former. In this regard, the mutant trachea showed cells that were less elongated and more "square-shaped" than wt ones (Figure 1, L and M). In addition, when the apical cell surface areas were measured, we could conclude that apical cell surface area in Blimp-1 mutants was reduced to about half of wt apical cell surface area (Figure $1 \mathrm{~N}$ ).

Taken together, these observations reveal that Blimp-1 affects various stages of tube maturation, from chitin deposition to tube expansion, as well cellular morphology.

\section{Blimp-1 modulates the timing of chitin deposition}

In wt embryos, a matrix composed of chitin and proteins such as Gasp, accumulates in the tracheal lumen. This matrix plays a key role in the regulation of tube length and diameter expansion (Moussian et al. 2005, 2015; Tonning et al. 2005). Due to the striking tube size phenotype of Blimp-1 mutants, and, because mutations in genes involved in chitin biogenesis and assembly result in irregular diametric expansion leading to locally constricted and dilated tubes, we examined the deposition of these markers from stage 12 (Figure 2). In the $w t$, early chitin deposition began at stage 13, when a chitinous filament started to be deposited inside the DT just prior to tube expansion (Moussian et al. 2015). In parallel, Gasp was detected from stage 13, but at this stage it was mainly cytoplasmic. None of these markers were detected at earlier stages. At stage 13, chitin began to be deposited in the lumen of all branches, starting from the DT (Moussian et al. 2015), and a mature pattern was achieved at stage 17, when taenidial ridges are fully formed (Tiklová et al. 2013; Öztürk-Çolak et al. 2016). Blimp-1 mutant embryos showed chitin deposition as early as stage 12 (Figure 2, B and C). The pattern of chitin deposition in mutants at stage 13 was similar to that of the wt (Figure 2, D and F). However, at later stages, the trachea of Blimp-1 mutants showed lower levels of chitin than the wt (Figure 2G) (Öztürk-Çolak et al. 2016). Gasp levels were lower in Blimp-1 mutants throughout embryogenesis, especially in the DT during later stages (Figure $2 \mathrm{~K}$ ), a pattern resembling that of embryos mutant for genes involved in chitin synthesis and organization (Araújo et al. 2005). We have previously shown that when we overexpressed Blimp-1 in the posterior part of the embryo using 

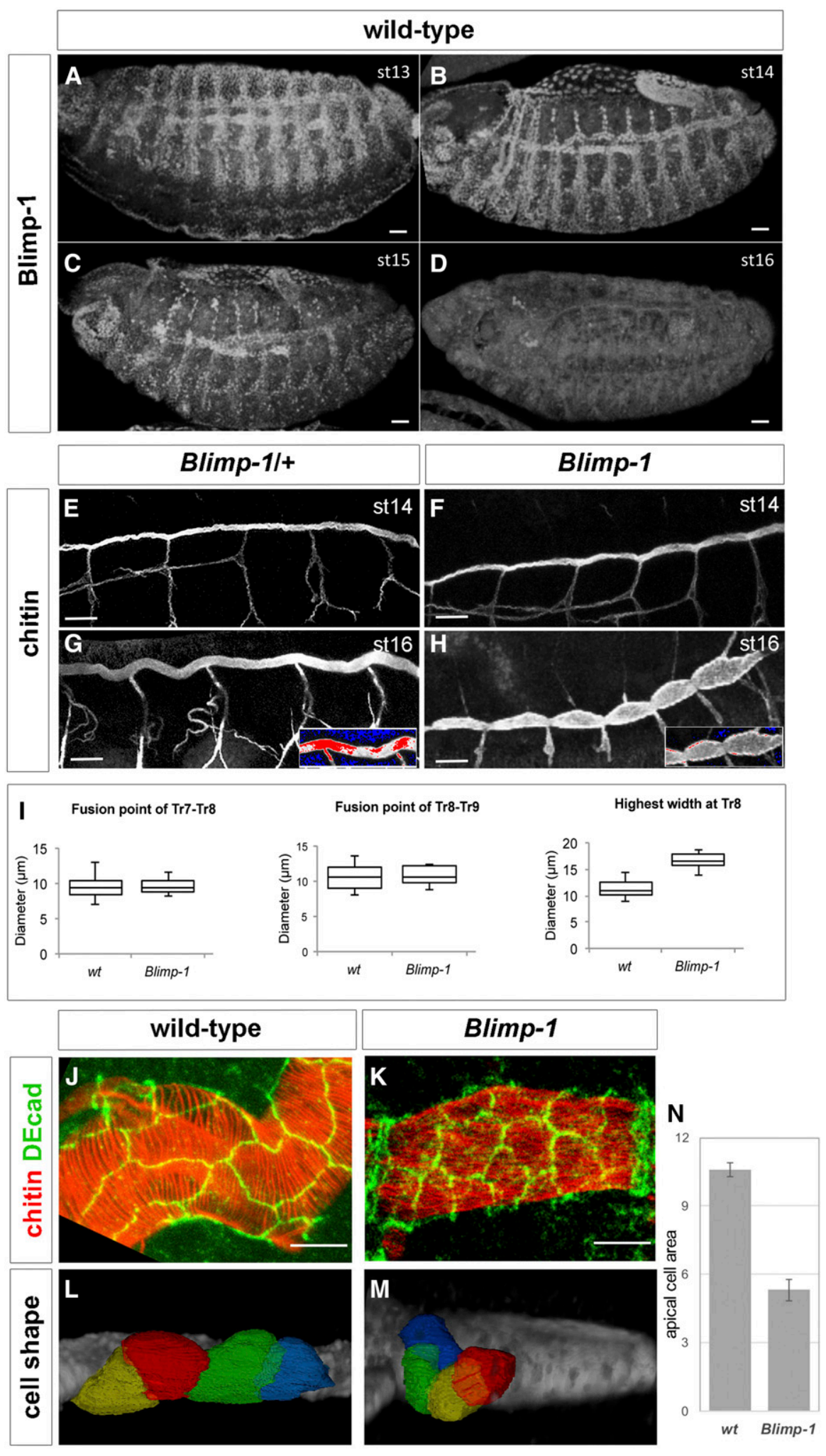

Figure 1 Blimp-1 is required for tracheal tube maturation. (A-D) Wild-type embryos stained for Blimp-1 protein with an anti-Blimp-1 antibody and showing Blimp-1 localization in tracheal cells from stage 13 to stage 15 . At stage 15 , Blimp-1 starts to be downregulated from the dorsal trunk (DT) and protein is not detected in tracheal cells at stage 16. Bar, $20 \mu \mathrm{m}$. (E-H) At stage 14, Blimp-1 tracheal DT is not distinguishable from the wild-type DT. Differences are observed at later stages of development, when Blimp-1 embryos display DTs with tube expansions between DT fusion points $(H)$. ( $G$ and $H)$ insets show detail of DT under a HiLo LUT, where highest intensity levels are red and lowest are blue, so differences in chitin amounts can be observed. Bar, $10 \mu \mathrm{m}$. (I) Quantification of tube width at fusion points and at between fusion points. (J-M) Blimp-1 DT tracheal cells have smaller apical domains than wild-type DT tracheal cells. Bar, $5 \mu \mathrm{m}$. (N) Quantification of apical cell surface area in wt and Blimp-1 DTs $(n=15, P$-value $<0.001)$.
Abd-BGAL4 to create tracheal DTs with distinct cellular compositions (Förster et al. 2009), we could detect lower levels of chitin in the posterior domain expressing higher levels of Blimp-1 (Öztürk-Çolak et al. 2016). To further analyze the influence of Blimp-1 in timely chitin deposition, we used the same experimental conditions and found that chitin was hardly detected in the posterior part of the trachea (Figure 2L) (Öztürk-Çolak et al. 2016). However, further examination with higher laser power and gain showed that indeed both the chitin filament and taenidia were present 


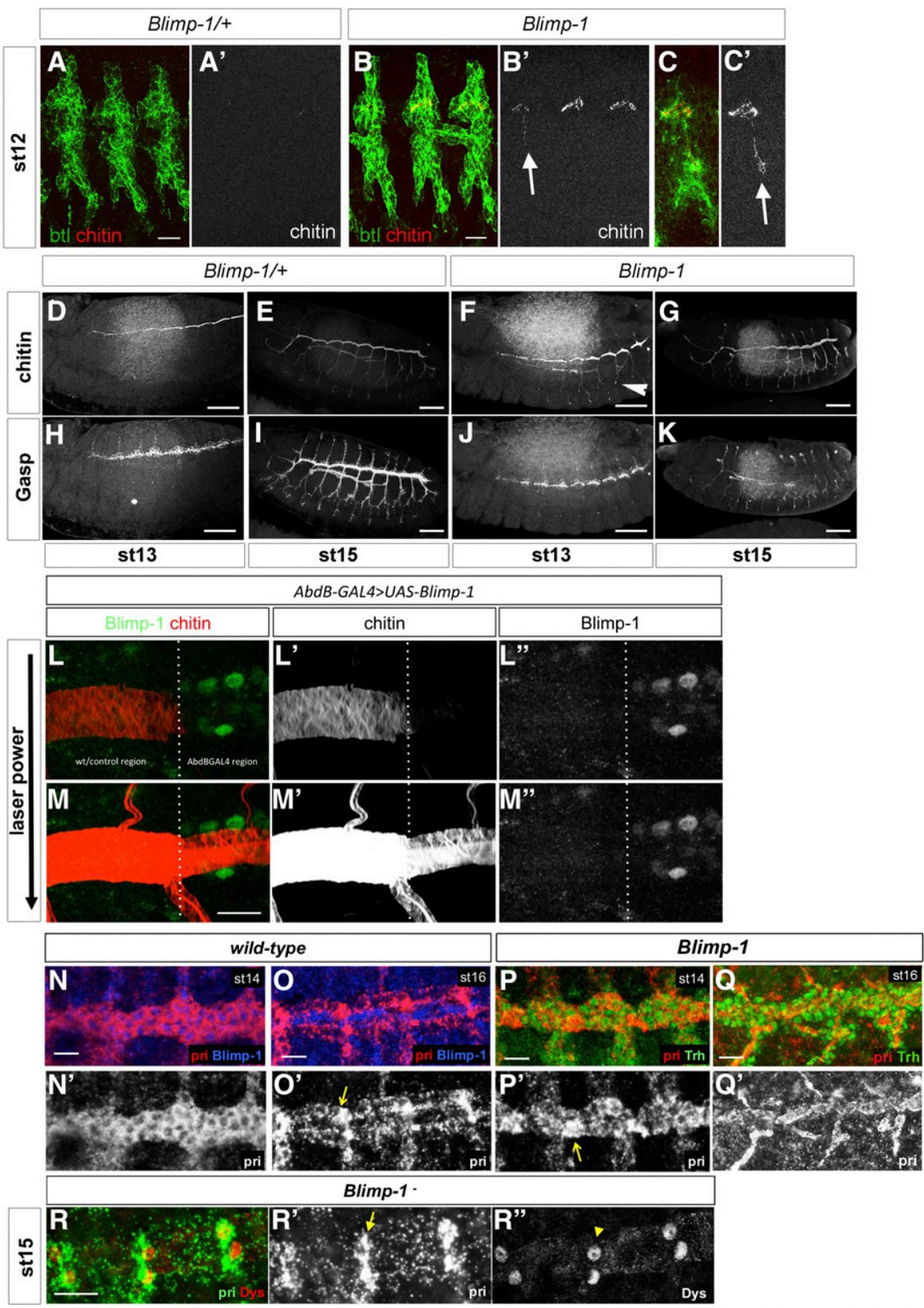

Figure 2 The timing of tube maturation is altered in Blimp-1 mutants. (A-C) The deposition of chitin in Blimp-1 embryos starts earlier than in the wild type and can be detected in the primordia of the DT and in some of the transverse connectives (LTs, arrows) from stage 12 ( $B, B^{\prime}$ and $C, C^{\prime}$ versus $\left.A, A^{\prime}\right)$. Btl positive cells are detected by a btl::moeGFP construct, stained with GFP. Bar, $5 \mu \mathrm{m}$. (D-K) Comparison of chitin and Gasp deposition in heterozygous ( $D, E, H$, and I) vs. homozygous ( $F, G, J$, and $K$ ) Blimp-1 embryos. Gasp deposition is defective in Blimp-1 embryos at stage 15, regardless of earlier chitin deposition. Bar, $40 \mu \mathrm{m}$. (L, $L^{\prime}, L^{\prime \prime}$ and $M, M^{\prime}$, $\left.M^{\prime \prime}\right)$ Detail of an early stage 17 DT at the border between cells expressing normal Blimp-1 levels and higher Blimp-1 levels driven by AbdBGAL4. Overexpression of Blimp-1 delays tube maturation, as reflected by lower chitin deposition. Bar, $10 \mu \mathrm{m}$. (N-P) Fluorescent in situ hybridization of pri (red) costained with either anti-Blimp-1 (blue) to label Blimp-1-expressing cells, or antiTrh (green) to label tracheal cells, in wild-type stage $14(\mathrm{~N})$ and stage $16(\mathrm{O})$ and Blimp-1 mutant stage $14(\mathrm{P})$ embryos. In wild-type embryos, at stage 14 pri is uniformly expressed throughout the tube $\left(\mathrm{N}^{\prime}\right)$. Later, at stage 16, pri expression starts to gradually disappear except at fusion points $\left(\mathrm{O}^{\prime}\right.$, arrow). In the Blimp-1 mutant embryo this differential expression of pri is already present at stage 14 . Note the lower levels of pri expression throughout the tube at stage 14 of the Blimp-1 mutant embryo $\left(P^{\prime}\right)$, except at the fusion points (arrow). (Q,Q') pri expression at stage 16 Blimp-1 mutant embryos. Bar, $10 \mu \mathrm{m}$. (QR) pri-expressing cells in Blimp-1 loss of function correspond to the fusion cells. Fluorescent in situ hybridization of pri (green) costained with anti-Dys (red) to label fusion cells in a Blimp-1 mutant embryo at stage 15 . The pri expression (arrow in $\mathrm{R}^{\prime}$ ) colocalizes with dys expression (arrowhead in $\mathrm{R}^{\prime \prime}$ ), indicating that the pri-expressing cells are fusion cells. throughout the trachea, although they displayed much lower levels in the posterior metameres, resembling chitin levels at earlier stages of development (Figure 2M). Blimp-1 overexpression lead to a tubular structure that regarding chitin composition and organization was apparently younger than the same tube with normal levels of Blimp-1 protein. Thus, Blimp-1 seems to regulate the timing of the beginning of chitin deposition by repressing target proteins. When Blimp-1 levels are high, chitin deposition is delayed, whereas, when they are low, chitin deposition begins earlier in the tracheal tubes.

\section{The expression pattern of tarsal-less/polished rice is altered in Blimp-1 mutants}

Mutants for tarsal-less (tal), also known as polished rice (pri), are affected in both F-actin and taenidia organization, and we analyzed them in parallel to Blimp-1 mutants (Öztürk-Çolak et al. 2016). Like Blimp-1, the tracheal expression of tal/pri in wild-type embryos is regulated by ecdysone (ChanutDelalande et al. 2014) (Figure S1) and started in tracheal cells at stage 12 (Figure S2). At early stages this expression was restricted to DT cells, but was later observed uniformly in all tracheal cells until stage 15 (Figure S2). From this stage, $\mathrm{tal} / \mathrm{pri}$ expression gradually decreased in all DT cells, except fusion cells, and, at stage 16, coinciding with the time Blimp1 disappears from tracheal cells, it became lower in almost all DT cells except in fusion cells (Figure $2 \mathrm{O}$ and Figure S2). tal/ pri expression in Blimp-1 mutants at stage 14 was not uniform in DT cells as in the $w t$. It was higher in fusion cells relative to the other DT cells (Figure 2P). To verify whether the cells with higher tal/pri expression in comparison to the 
A

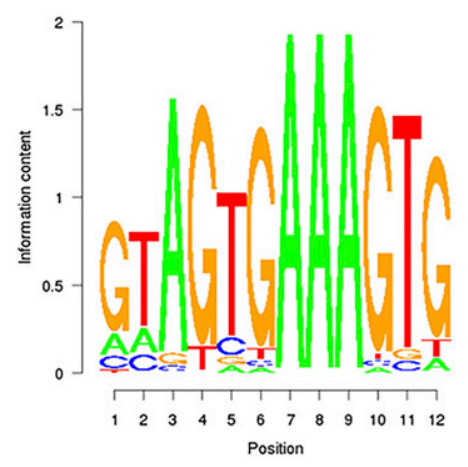

B

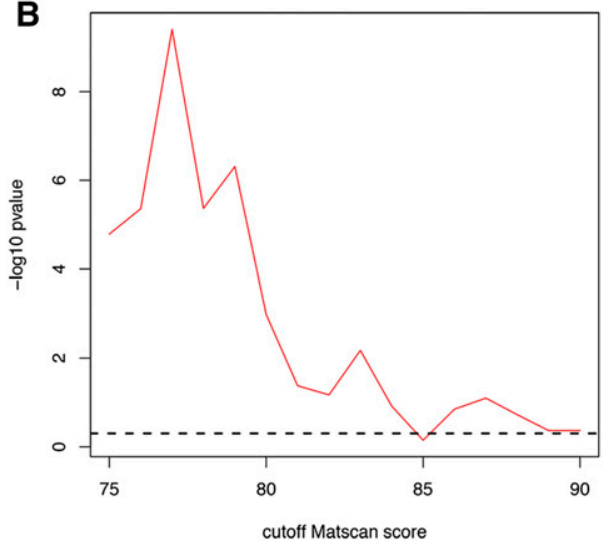

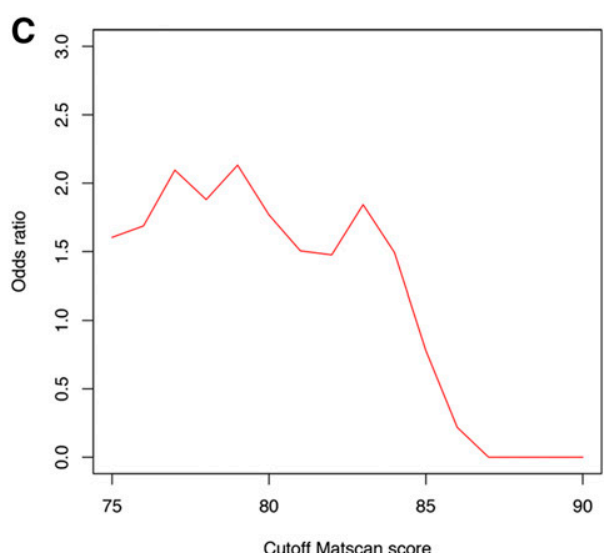

Figure 3 Blimp-1 position weight matrix. (A) Position weight matrix used in this study, which was taken from the reported binding sequences in Kuo and Calame (2004). (B and C) Enrichment analysis of known tracheal genes among genes predicted to be regulated by Blimp-1. P-values and ORs are shown for Fisher tests with varying Matscan scores for the definition of predicted binding sites. For values $<85$, ORs are relatively constant and $>1.5$ (associated $P$-values $<0.05$, dashed line) showing robustness against variations of the Matscan parameter.

other DT cells were indeed fusion cells, we double labeled the embryos with the fusion cell marker dysfusion (dys) (Jiang and Crews 2003). Higher levels of both tal/pri and dys were detected in the same cells, thereby confirming that these are indeed fusion cells (Figure 2Q). These results indicate that tal/pri expression at stage 14 Blimp-1 embryos resembles expression of tal/pri in stage $16 \mathrm{wt}$ embryos, suggesting that Blimp-1 regulates the timing of the onset of tal/pri differential expression, from embryonic stage 16 . We hypothesize that Blimp-1 might be involved in the differential regulation of tal/pri expression in tracheal cells. When Blimp-1 levels are low, tal/pri expression levels go down in DT, and are maintained at high levels only in fusion cells. We speculate that Blimp-1 could play a role in repressing tal/pri expression at high levels in fusion cells and, from stage 16, when Blimp-1 is no longer present in tracheal cells, this repression would no longer be exerted, leading to higher levels of expression in fusion cells.

\section{Blimp-1 regulates a variety of genes involved in tube maturation}

To further study the influence of Blimp-1 in tracheal development, we searched in silico for Blimp-1 binding sites in the promoter regions of all $D$. melanogaster genes using the Matscan software (Blanco et al. 2006) and the reported position weight matrix corresponding to Blimp-1 (Kuo and Calame 2004). We found 3949 genes (6332 different isoforms) with at least one putative binding site (binding score $>75 \%$ of maximum value) within 2000 bases of their annotated TSSs. We prioritized candidate positions on the basis of binding score and evolutionary conservation from the UCSC Drosophila-related species track (Fujita et al. 2011) (Table S1). In particular, we found that Blimp-1 can potentially regulate its own transcription, and also the transcription of a variety of genes involved in tracheal tube development. We asked whether Blimp-1 regulation of tracheal genes was enriched in relation to all genes in the fly genome. The transcription factor Trachealess (Trh) is among the first genes to be expressed in the cells that will form the trachea. In the absence of Trh, tracheal cells fail to invaginate to form tubes and remain at the embryo surface (Isaac and Andrew 1996; Wilk et al. 1996). It has been shown that expression of nearly every tracheal gene requires trh (Chung et al. 2011). Thus, we wondered how many genes regulated by Blimp-1 were also downstream of Trh, and, therefore, bona fide tracheal genes. To test this, we combined published tracheal gene sets (Chung et al. 2011; Hammonds et al. 2013) and checked for enrichment among the genes with predicted Blimp-1 binding sites. We found that for a conservation threshold of 2 and Matscan scores $>75 \%$, tracheal genes are significantly enriched [Fisher-test odds ratio (OR) $=$ $1.60, P$-value $<0.001]$. In order to show robustness against the choice of Matscan score threshold, we repeated the Fisher test for values ranging from 75 to $90 \%$. We found that the OR was kept relatively constant until $84 \%$, dropping to nonsignificant values for the remaining thresholds (Figure 3, B and $\mathrm{C}$ and Table S3).

We then selected a shorter list of genes reported to be involved in tube maturation stages (Table 1). Clearly, this is not an exhaustive list as it includes only the genes detected using these restrictive parameters. Thus, for example, this analysis did not allow us to detect any Blimp- 1 binding sites in the tal/pri region. However, due to the changes in tal/pri expression in Blimp-1 mutants, we further studied the tal/pri region under less restrictive conditions. We detected four binding sites with Matscan scores above the 72 percentile and conservation scores of 1 .

\section{Blimp-1 regulates the levels of Exp, Reb, aPKC, Knk, and Btk29A}

In order to further analyze the relationship between Blimp-1 and tracheal maturation, we compared the levels of five key proteins, Exp, Reb, aPKC, Knk, and Btk29A, in tube maturation in the heterozygous and homozygous Blimp-1 mutant 
Table 1 Possible downstream targets of Blimp-1 known to be involved in tracheal tube maturation

\begin{tabular}{lll}
\hline Gene & \multicolumn{1}{c}{ Function } & Reference \\
\hline atypical Protein Kinase C (aPKC) & Member of the Par complex & Hosono et al. (2015) \\
bitesize (btsz) & Synaptotagmin-like protein & Jayanandanan et al. (2014) \\
Blimp-1 & Transcription factor & Chavoshi et al. (2010) \\
Btk family kinase at 29A (Btk29A) & Nonreceptor tyrosine kinase & Tsikala et al. (2014) \\
expansion (exp) & Required for chitin deposition & Moussian et al. (2015) \\
gartenzwerg (garz) & Secretion & Chung et al. (2011) \\
grainy-head (grh) & Transcription factor & Hemphälä et al. (2003) \\
knickhopf (knk) & Chitin organization & Chung et al. (2011) \\
obstructor-A (obst-A) & Chitin-binding protein & Chung et al. (2011) \\
pebbled (peb) & Transcription factor & Wilk et al. (2000) \\
rebuf (reb) & Required for chitin deposition & Moussian et al. (2015) \\
shade (shd) & Ecdysone pathway enzyme & Chavoshi et al. (2010) \\
sinuous (sinu) & Septate junction component & Wu et al. (2004) \\
tramtrack (ttk) & Transcription factor & Araújo et al. (2007) \\
\hline
\end{tabular}

Matscan score within the upper 75 percentile (and conservation score $>2$ ).

embryos. Exp and Reb are atypical Smad-like proteins that regulate tube size in the tracheal system by promoting chitin deposition (Moussian et al. 2015). aPKC is a serine/threonine protein kinase required for apico-basal cell polarity and a member of the Par complex. It has been shown to be involved in the orientation of actin rings and taenidial ridges in larval stages of tube maturation (Hosono et al. 2015). Knk is a GPI anchored protein needed for chitin organization and the regulation of tracheal tube diameter (Moussian et al. 2006). Btk29A (also known as Tec29A) is the only member of the Tec family of kinases in Drosophila, and it is expressed in many developmental stages of the fly. In the tracheal system, Btk29A is involved in spiracular chamber invagination, as well as in tracheal cuticle patterning (Matusek et al. 2006; Tsikala et al. 2014). Mutants and overexpression conditions for each of these genes showed phenotypes that can be correlated to the Blimp-1 tracheal maturation phenotypes. We therefore hypothesized that Blimp-1 acts as a transcriptional repressor of these genes during tube maturation (Matusek et al. 2006; Moussian et al. 2006, 2015; Hosono et al. 2015).

At early tube maturation stages, Blimp-1 mutants displayed higher levels of Exp, aPKC, Knk, and Btk29A than same-stage $w t$ embryos (Figure 4), thereby suggesting that Blimp-1 represses the expression of these proteins, as hypothesized. Of the four proteins studied, Btk29A levels showed a more pronounced difference between heterozygous and homozygous Blimp-1 mutant embryos. Btk29A levels at stage 14 were hardly detectable in the tracheal system of wt embryos (Figure 4G') (Tsikala et al. 2014), whereas, in mutant tracheal cells, they were detected at much higher levels (Figure 4H'). Taken together with our previous in silico approach, our findings suggest that Blimp-1 directly regulates the levels of these five proteins in tracheal cells during tube maturation, working as a repressor during early tracheal developmental stages.

During lumen maturation, chitin deposition requires the activity of Krotzkopf verkehrt (Kkv) together with Exp and Reb (Moussian et al. 2015). However, we could not find any evidence that Blimp-1 regulates $k k v$ expression. Due to the earlier chitin deposition observed in Blimp-1 mutant embryos (Figure 2), we wondered if Reb expression was also detected earlier, consequently leading to earlier chitin deposition. Indeed, we observed that in Blimp-1 homozygous embryos, Reb expression was already detected at stage 12, in contrast to wild-type and Blimp-1 heterozygous embryos, where it starts being detected at stage 13 (Figure 4, I-L) (Moussian et al. 2015). Thus, the earlier chitin deposition detected in Blimp-1 embryos may be triggered by this earlier expression of Reb together with the higher levels of Exp in tracheal cells.

\section{Btk29A works downstream of Blimp-1 to regulate luminal aECM organization}

What is the role of Btk29A in tube maturation downstream of Blimp-1? At late embryonic stages, a strong Btk29A mutant allele displays disorganized apical F-actin bundles and taenidial ridges (Matusek et al. 2006; Öztürk-Çolak et al. 2016), with heterogeneous patterns of F-actin bundling in the same trachea, showing stretches of perpendicular bundles followed by stretches of parallel bundles (Öztürk-Çolak et al. 2016). In addition, overexpression of full-length Btk29A in all tracheal cells gave rise to an expansion phenotype similar to Blimp-1 mutants at stage 16 (Figure 5C, cf. Figure 1H). Thus, and due to our hypothesis of Blimp-1 being a repressor of Btk29A expression, we examined whether derepression of Btk29A might partially account for the Blimp-1 mutant phenotype. To do so, we used Btk29A ${ }^{K 00206}$, a hypomorphic allele in which low mRNA levels are still detected in the embryonic tracheal system (Tsikala et al. 2014). In this hypomorphic allele, there were no apparent differences in chitin deposition between heterozygous and homozygous Btk29A mutant embryos at stage 16 (Figure 5, A and B). At late stage 17, Btk29A K00206 homozygous embryos showed mild DT phenotypes compared to heterozygous embryos, such as a very mild DT expansion phenotype (Figure 5, D and $\mathrm{E})$. However, there were no detectable taenidial ridge orientation phenotypes in stage $17 \mathrm{Btk} 29 \mathrm{~A}^{\text {K00206 }}$ mutants, 


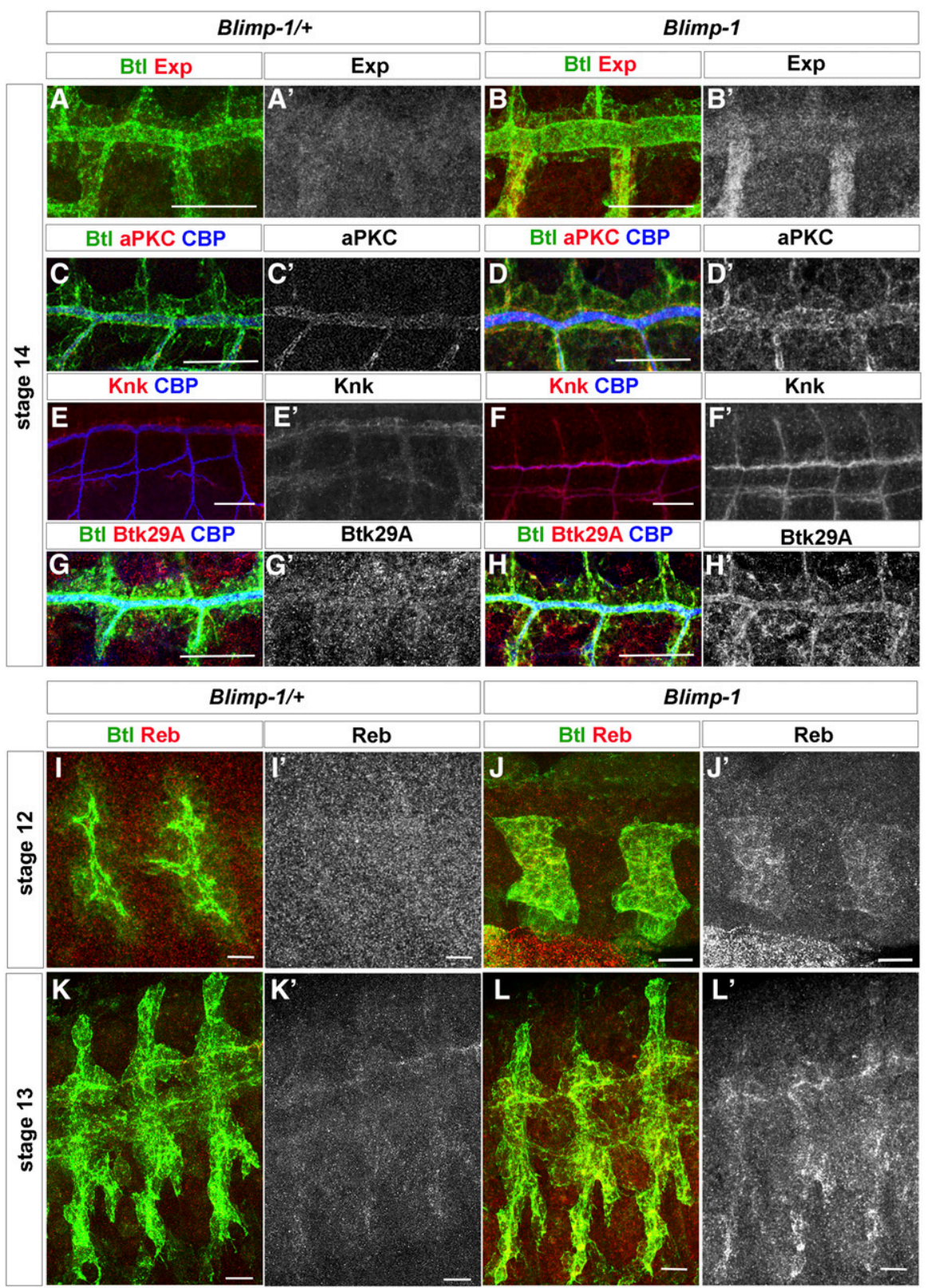

Figure 4 Blimp-1 regulates the expression of genes involved in tube maturation. $\left(A, A^{\prime}\right.$ and $\left.B, B^{\prime}\right) \operatorname{Exp},\left(C, C^{\prime}\right.$ and $\left.D, D^{\prime}\right)$ aPKC, $\left(E, E^{\prime}\right.$ and $F$, $\left.F^{\prime}\right)$ Knk and $\left(G, G^{\prime}\right.$ and $\left.H, H^{\prime}\right)$ Btk29A levels are higher in Blimp-1 mutants than in the wild-type at stage 14. Bar, $20 \mu \mathrm{m}$. (I-L) Reb expression starts earlier in Blimp-1 mutant embryos; it is already detected in stage 12 mutants whereas it starts at stage 13 in heterozygous Blimp-1 embryos (I, I' and J, J'); heterozygous stage $12 \mathrm{em}$ bryos in (I) are older than mutant embryos in (J) reinforcing the earlier expression of Reb in Blimp-1 embryos. ( $K, \mathrm{~K}^{\prime}$ and $\left.\mathrm{L}, \mathrm{L}^{\prime}\right)$ Reb expression is higher in stage 13 Blimp-1 mutant embryos. Bar, $10 \mu \mathrm{m}$. which showed parallel taenidial ridges perpendicular to the tube length as in the wild-type (Figure 5, E and E'). We then combined the Btk29A $\mathrm{A}^{\text {Koo206 }}$ mutation with Blimp-1 and observed that the incomplete removal of embryonic Btk29A could partially rescue the taenidial ridge orientation phenotypes in most of the embryos examined $(64 \%, n=11)$. These double Btk29AK00206; Blimp-1 mutants showed heterogeneous patterns of taenidial ridge organization in the same trachea, showing stretches of perpendicular bundles followed by stretches of parallel ones (45\% of embryos, Figure $5, \mathrm{G}$ and $\mathrm{H}$, arrow in $\mathrm{G})$, as well as diagonal ridges (19\% of embryos, Figure 5H, arrow). We also observed that Btk29A; Blimp-1 double mutant DTs showed a milder tube expansion phenotype than Blimp-1 mutants (Figure 5, F-H). Taken together, these results indicate that part of the Blimp-1 phenotype can be attributed to excess Btk29A.

\section{Discussion}

Here, we found that Blimp-1 regulates multiple tracheal targets, thus acting as a key gene in tracheal development (Figure 6A and Table 1). Blimp-1 is an ecdysone response gene (Beckstead et al. 2005; Chavoshi et al. 2010) (Figure S1) and, therefore, a link between the hormonal signal and the timing of tracheal tube maturation in both embryos and larvae. We show that Blimp-1 regulates the expression of many genes required for tube maturation. Interestingly, in silico, we detected four Blimp-1 binding sites in Blimp-1 regulatory sequences using the parameters described, which suggests that Blimp-1 may regulate its own expression. This is in agreement with recent data showing that Blimp-1/PRDM1 is also able to regulate its own expression in mammals (Mitani et al. 2017). Self-regulation of expression is consistent with 


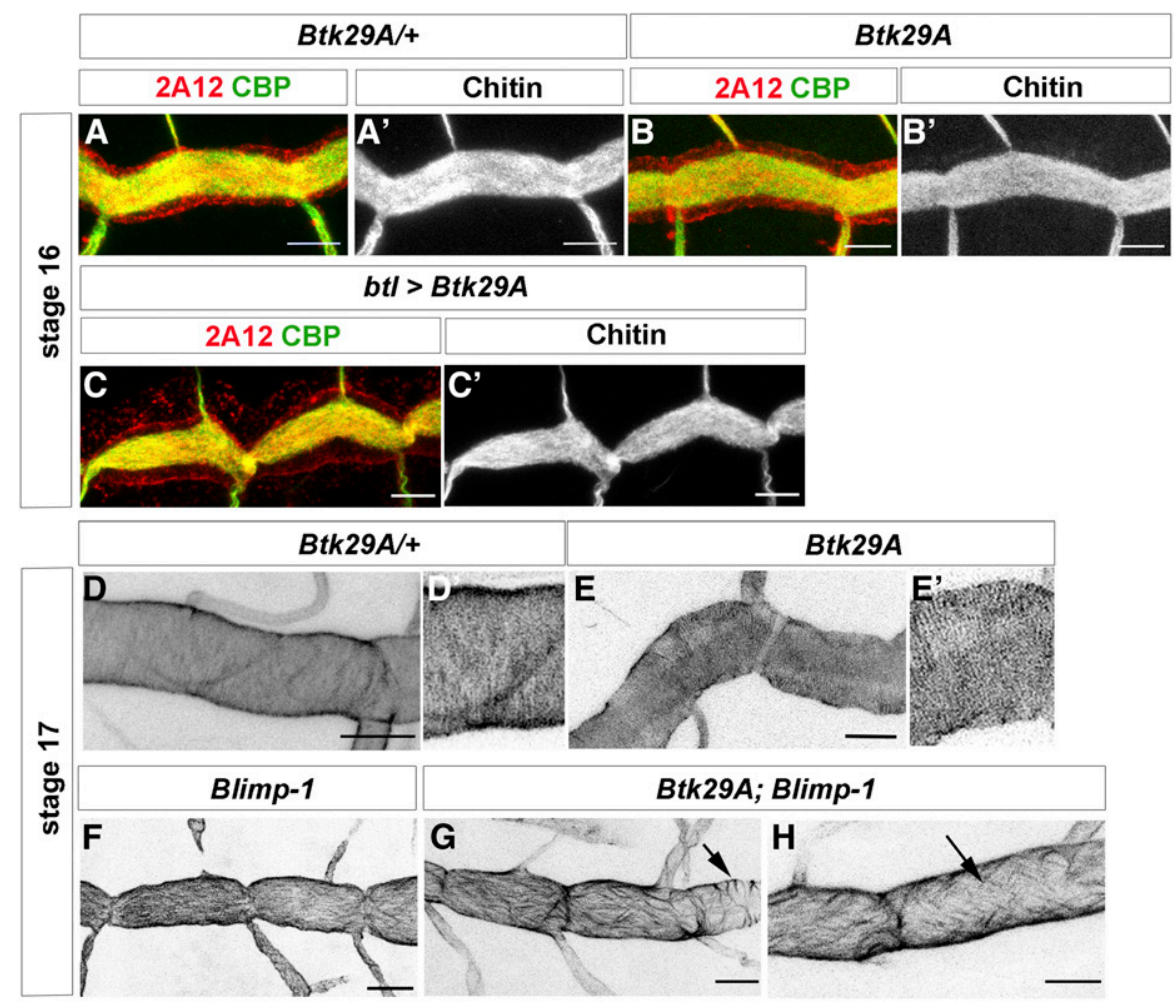

Figure 5 Btk29A partially rescues Blimp-1 phenotypes. ( $A, A^{\prime}$ and $\left.B, B^{\prime}\right) B t k 29 A^{K 00206}$ embryos do not show any strong tube maturation phenotypes at late embryonic stages. (C, $C^{\prime}$ and $D$, D') Btk29A overexpression in tracheal cells induces tracheal phenotypes similar to Blimp-1 mutant embryos. (D-H) At the end of embryogenesis, stage 17 , taenidial ridges can be detected by staining with chitin binding probes (CBP) (D and D'); Btk29A ${ }^{K 00206}$ embryos display mild taenidial ridge phenotypes ( $E$ and $E^{\prime}$ ); however, when in a Blimp-1 mutant background, a Btk29A hypomorphic mutation such as the one present in the K00206 allele, partially rescues the Blimp-1 expansion and taenidial ridge phenotypes $(G$ and $H)$. Bar, $5 \mu \mathrm{m}$ in all panels. the feedback loops in which Blimp-1/PRDM1 participates, and also with its role in regulating many developmental processes (Gong and Malek 2007; Bikoff et al. 2009). We also found Blimp-1 binding sites in the region of Tramtrack (Ttk), another transcription factor involved in many features of tube maturation (Araújo et al. 2007).

Furthermore, we observed that Blimp-1 modulates the timing of the expression of $r e b$ and exp, two genes involved in the genetic program triggering timely chitin deposition
(Moussian et al. 2015). Untimely chitin deposition was shown to disturb tube maturation, thereby demonstrating that this process has to be tightly regulated during tracheal development. Tracheal overexpression of reb leads to earlier chitin deposition in all branches from stage 13 and sometimes chitin appearance at stage 12 (Moussian et al. 2015; and M. Llimargas, personal communication). Accordingly, our results show that Reb is expressed earlier in Blimp-1 embryos (Figure 4, J and L). This agrees with the early chitin deposition phenotypes observed in
A

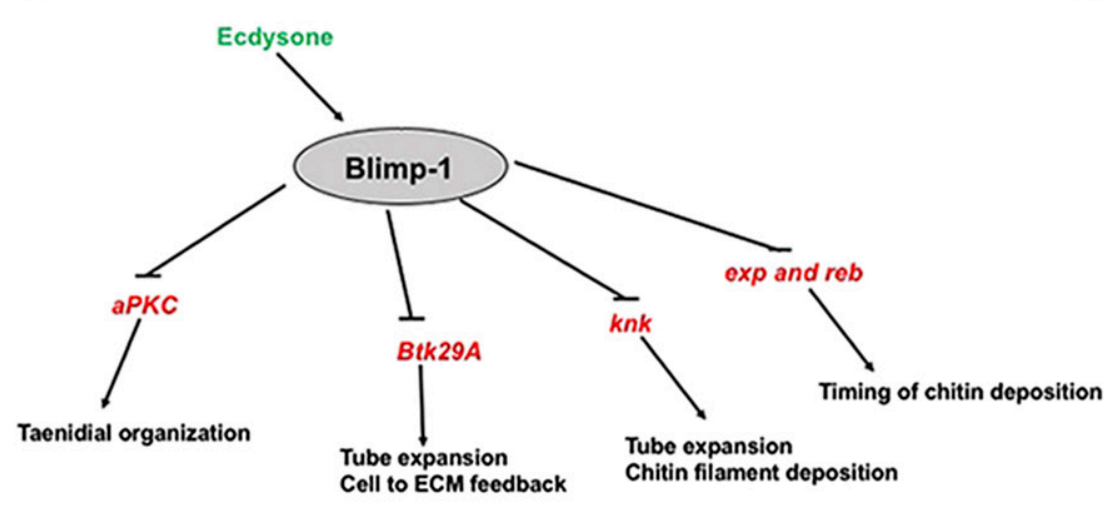

B

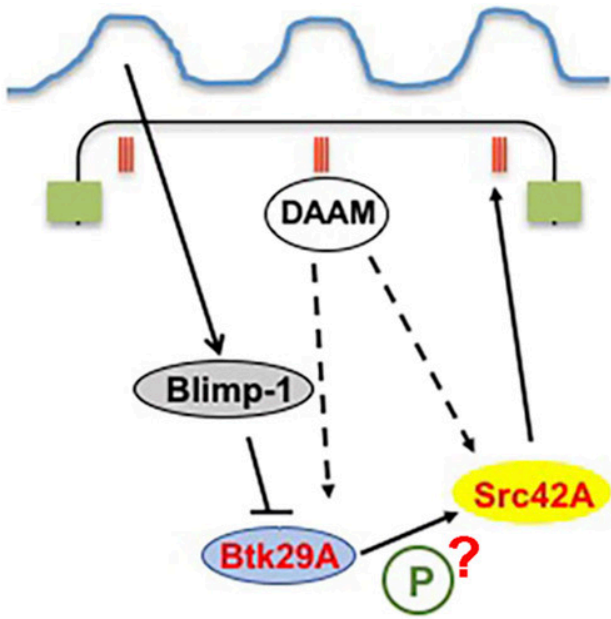

Figure 6 A model for the involvement of Blimp-1 in tracheal tube maturation timing. (A) Under the influence of the insect hormone ecdysone, Blimp-1 regulates tracheal tube maturation timing by inhibiting the expression of the key genes for tube maturation, among them aPKC, Btk29A, knk, exp, and $r e b$. Some of these targets are known to be activated by other transcription factors, like, for example, Ttk in the case of Exp, Spalt (Sal) in the case of Reb, and Trh in the case of Knk (Chung et al. 2011; Moussian et al. 2015). (B) Blimp-1 affects taenidial ridge formation by acting as a transcriptional repressor of Btk29A, which is an important molecule for Src42A phosphorylation. Phosphorylated Src42A and the formin DAAM regulate the actin cytoskeleton inside the cells, hence giving rise to taenidial ridges at the extracellular matrix. 
Blimp-1 mutants (Figure 2, B and C). Furthermore, Blimp-1 also modulated $k n k$ expression during tube maturation stages. Knk is involved in directing chitin assembly in the trachea (Moussian et al. 2006) and correct amounts of Knk at specific times during metamorphosis are important for correct wing cuticle differentiation and function ( $\mathrm{Li}$ et al. 2017). Taken together, these in silico and in vivo results indicate that Blimp-1 is a transcription factor that acts downstream of ecdysone, and that it is involved in the correct timing of chitin synthesis and deposition during embryonic development.

We also found Blimp-1 binding sites in the aPKC coding region. aPKC is involved in the junction anisotropies that orient both actin rings and taenidial ridges in the lumen of tracheal tubes (Hosono et al. 2015). In Blimp-1 mutants, both actin rings and taenidial ridges are either undetectable or misoriented (Öztürk-Çolak et al. 2016)_observations that are consistent with changes in junction anisotropy.

We previously showed that Blimp-1 regulates chitin deposition levels and architecture, and that, subsequently, the chitin aECM feeds back on the cellular architecture by stabilizing F-actin bundling and cell shape via the modulation of Src42A phosphorylation levels (Öztürk-Çolak et al. 2016). However, in this report, we provided no link between the chitinous aECM and Src42A. Btk29A mutant larvae have an aECM phenotype, which may be the result of their actin bundle phenotype (Matusek et al. 2006; Öztürk-Çolak et al. 2016). Here, we found that Btk29A removal can partially rescue the Blimp-1 taenidial orientation and expansion phenotype. In view of these results, we propose that the contribution of Btk29A can be added to the feedback model for the generation of supracellular taenidia put forward in ÖztürkÇolak et al. (2016). We now add to our previous model by hypothesizing that Blimp-1 acts as a link between the aECM and cells by regulating the levels of Btk29A (Figure 6B). Btk29A and Src42A, together with the formin DAAM, have been shown to regulate the actin cytoskeleton (Matusek et al. 2006). In agreement with our results, we speculate that Btk29A might phosphorylate Src42A, and that this phosphorylation event could be modulated by Blimp-1 and DAAM.

To conclude, our results indicate that Blimp-1 is a key player in the regulation of tracheal tube maturation, and, consequently, in the feedback mechanism involved in the generation of supracellular taenidia.

\section{Acknowledgments}

We thank M. Llimargas, E. Sanchez-Herrero, S. Roy, H. Ueda, and the Bloomington Stock Center for flies and reagents. Thanks also go to the Institut de Recerca Biomedica de Barcelona (IRB Barcelona) Biostatistics and Bioinformatics Unit. We acknowledge L. Bardia, A. Lladó, and J. Colombelli from the IRB Barcelona Advanced Digital Microscopy Facility for help and advice with confocal microscopy and software, and E. Fuentes and N. Martin for technical assistance. S.J.A. was a Ramon y Cajal Researcher (RYC-2007-00417); A.O. was the recipient of an IRB-La
Caixa fellowship. This work was supported by grants from the Generalitat de Catalunya and the Spanish Ministerio de Ciencia e Innovación (BFU2009-07629).

\section{Literature Cited}

Agawa, Y., M. Sarhan, Y. Kageyama, K. Akagi, M. Takai et al., 2007 Drosophila Blimp-1 is a transient transcriptional repressor that controls timing of the ecdysone-induced developmental pathway. Mol. Cell. Biol. 27: 8739-8747. https://doi.org/10. 1128/MCB.01304-07

Akagi, K., M. Sarhan, A.-R. S. Sultan, H. Nishida, A. Koie et al., 2016 A biological timer in the fat body comprising Blimp-1, $\beta F t z-f 1$ and Shade regulates pupation timing in Drosophila melanogaster. Development 143: 2410-2416. https://doi.org/10. 1242/dev.133595

Araújo, S. J., H. Aslam, G. Tear, and J. Casanova, 2005 Mummy/ cystic encodes an enzyme required for chitin and glycan synthesis, involved in trachea, embryonic cuticle and CNS developmentanalysis of its role in Drosophila tracheal morphogenesis. Dev. Biol. 288: 179-193. https://doi.org/10.1016/j.ydbio.2005.09.031

Araújo, S. J., C. Cela, and M. Llimargas, 2007 Tramtrack regulates different morphogenetic events during Drosophila tracheal development. Development 134: 3665-3676. https://doi.org/ 10.1242/dev.007328

Beckstead, R. B., G. Lam, and C. S. Thummel, 2005 The genomic response to 20-hydroxyecdysone at the onset of Drosophila metamorphosis. Genome Biol. 6: R99. https://doi.org/10.1186/ gb-2005-6-12-r99

Bikoff, E. K., M. A. Morgan, and E. J. Robertson, 2009 An expanding job description for Blimp-1/PRDM1. Curr. Opin. Genet. Dev. 19: 379-385. https://doi.org/10.1016/j.gde.2009.05.005

Blanco, E., X. Messeguer, T. F. Smith, and R. Guigo, 2006 Transcription factor map alignment of promoter regions. PLOS Comput. Biol. 2: e49. https://doi.org/10.1371/journal.pcbi.0020049

Campos-Ortega, A. J., and V. Hartenstein, 1985 The Embryonic Development of Drosophila Melanogaster, pp. 10-84. SpringerVerlag, New York. https://doi.org/10.1007/978-3-662-02454-6

Chanut-Delalande, H., Y. Hashimoto, A. Pelissier-Monier, R. Spokony, A. Dib et al., 2014 Pri peptides are mediators of ecdysone for the temporal control of development. Nat. Cell Biol. 16: 1035-1044. https://doi.org/10.1038/ncb3052

Chavoshi, T. M., B. Moussian, and A. Uv, 2010 Tissue-autonomous EcR functions are required for concurrent organ morphogenesis in the Drosophila embryo. Mech. Dev. 127: 308-319. https://doi. org $/ 10.1016 /$ j.mod.2010.01.003

Chung, S., C. Chavez, and D. J. Andrew, 2011 Trachealess (Trh) regulates all tracheal genes during Drosophila embryogenesis. Dev. Biol. 360: 160-172. https://doi.org/10.1016/j.ydbio.2011.09.014

Cunningham, F., M. R. Amode, D. Barrell, K. Beal, K. Billis et al., 2015 Ensembl 2015. Nucleic Acids Res. 43: D662-D669. https://doi.org/10.1093/nar/gku1010

Fog, C. K., G. G. Galli, and A. H. Lund, 2012 PRDM proteins: important players in differentiation and disease. BioEssays 34 : 50-60. https://doi.org/10.1002/bies.201100107

Förster, D., K. Armbruster, and S. Luschnig, 2009 Sec24-dependent secretion drives cell-autonomous expansion of tracheal tubes in Drosophila. Curr. Biol. 20: 62-68. https://doi.org/10.1016/j. cub.2009.11.062

Fujita, P. A., B. Rhead, A. S. Zweig, A. S. Hinrichs, D. Karolchik et al., 2011 The UCSC genome browser database: update 2011. Nucleic Acids Res. 39: D876-D882. https://doi.org/10. 1093/nar/gkq963

Gong, D., and T. R. Malek, 2007 Cytokine-dependent Blimp-1 expression in activated $\mathrm{T}$ cells inhibits IL-2 production. 
J. Immunol. 178: 242-252. https://doi.org/10.4049/jimmunol. 178.1.242

Hammonds, A. S., C. A. Bristow, W. W. Fisher, R. Weiszmann, S. Wu et al., 2013 Spatial expression of transcription factors in Drosophila embryonic organ development. Genome Biol. 14: R140. https://doi.org/10.1186/gb-2013-14-12-r140

Hemphälä, J., A. Uv, R. Cantera, S. Bray, and C. Samakovlis, 2003 Grainy head controls apical membrane growth and tube elongation in response to Branchless/FGF signalling. Development 130: 249-258. https://doi.org/10.1242/dev.00218

Hohenauer, T., and A. W. Moore, 2012 The Prdm family: expanding roles in stem cells and development. Development 139: 2267-2282. https://doi.org/10.1242/dev.070110

Hosono, C., R. Matsuda, B. Adryan, and C. Samakovlis, 2015 Transient junction anisotropies orient annular cell polarization in the Drosophila airway tubes. Nat. Cell Biol. 17: 1569-1576. https://doi. org/10.1038/ncb3267

Huang, S., 1994 Blimp-1 is the murine homolog of the human transcriptional repressor PRDI-BF1. Cell 78: 9. https://doi. org/10.1016/0092-8674(94)90565-7

Isaac, D. D., and D. J. Andrew, 1996 Tubulogenesis in Drosophila: a requirement for the trachealess gene product. Genes Dev. 10: 103-117. https://doi.org/10.1101/gad.10.1.103

JayaNandanan, N., R. Mathew, and M. Leptin, 2014 Guidance of subcellular tubulogenesis by actin under the control of a synaptotagmin-like protein and Moesin. Nat. Commun. 5: 3036. https://doi.org/10.1038/ncomms4036

Jiang, L., and S. T. Crews, 2003 The Drosophila dysfusion basic helix-loop-helix (bHLH)-PAS gene controls tracheal fusion and levels of the trachealess bHLH-PAS protein. Mol. Cell. Biol. 23: 5625-5637. https://doi.org/10.1128/MCB.23.16.5625-5637.2003

Keller, A. D., and T. Maniatis, 1991 Identification and characterization of a novel repressor of beta-interferon gene expression. Genes Dev. 5: 868-879. https://doi.org/10.1101/gad.5.5.868

Kuo, T. C., and K. L. Calame, 2004 B lymphocyte-induced maturation protein (Blimp)-1, IFN regulatory factor (IRF)-1, and IRF2 can bind to the same regulatory sites. J. Immunol. 173: 55565563. https://doi.org/10.4049/jimmunol.173.9.5556

Li, K., X. Zhang, Y. Zuo, W. Liu, J. Zhang et al., 2017 Timed Knickkopf function is essential for wing cuticle formation in Drosophila melanogaster. Insect Biochem. Mol. Biol. 89: 1-10. https://doi.org/10.1016/j.ibmb.2017.08.003

Matusek, T., A. Djiane, F. Jankovics, D. Brunner, M. Mlodzik et al., 2006 The Drosophila formin DAAM regulates the tracheal cuticle pattern through organizing the actin cytoskeleton. Development 133: 957-966. https://doi.org/10.1242/dev.02266

Mitani, T., Y. Yabuta, H. Ohta, T. Nakamura, C. Yamashiro et al., 2017 Principles for the regulation of multiple developmental pathways by a versatile transcriptional factor, BLIMP1. Nucleic Acids Res. 45: 12152-12169. https://doi.org/10.1093/nar/ gkx798

Moussian, B., H. Schwarz, S. Bartoszewski, and C. Nusslein-Volhard, 2005 Involvement of chitin in exoskeleton morphogenesis in Drosophila melanogaster. J. Morphol. 264: 117-130. https:// doi.org/10.1002/jmor.10324

Moussian, B., E. Tång, A. Tonning, S. Helms, H. Schwarz et al., 2006 Drosophila Knickkopf and Retroactive are needed for epithelial tube growth and cuticle differentiation through their specific requirement for chitin filament organization. Development 133: 163-171. https://doi.org/10.1242/dev.02177

Moussian, B., A. Letizia, G. Martínez-Corrales, B. Rotstein, A. Casali et al., 2015 Deciphering the genetic programme triggering timely and spatially-regulated chitin deposition. PLoS Genet. 11: e1004939 (erratum: PLoS Genet. 11: e1005054). https:// doi.org/10.1371/journal.pgen.1004939

Ng, T., F. Yu, and S. Roy, 2006 A homologue of the vertebrate SET domain and zinc finger protein Blimp-1 regulates terminal differentiation of the tracheal system in the Drosophila embryo. Dev. Genes Evol. 216: 243-252. https://doi.org/10.1007/ s00427-005-0044-5

Öztürk-Çolak, A., B. Moussian, S. Araujo, and J. Casanova, 2016 A feedback mechanism converts individual cell features into a supracellular ECM structure in Drosophila trachea. eLife 5: e09373. https://doi.org/10.7554/eLife.09373

Schindelin, J., I. Arganda-Carreras, E. Frise, V. Kaynig, M. Longair et al., 2012 Fiji: an open-source platform for biological-image analysis. Nat. Methods 9: 676-682. https://doi.org/10.1038/ nmeth.2019

Smedley, D., S. Haider, S. Durinck, L. Pandini, P. Provero et al., 2015 The BioMart community portal: an innovative alternative to large, centralized data repositories. Nucleic Acids Res. 43: W589-W598. https://doi.org/10.1093/nar/gkv350

Tiklová, K., V. Tsarouhas, and C. Samakovlis, 2013 Control of airway tube diameter and integrity by secreted chitin-binding proteins in Drosophila. PLoS One 8: e67415. https://doi.org/ 10.1371/journal.pone.0067415

Tonning, A., J. Hemphälä, E. Tång, U. Nannmark, C. Samakovlis et al., 2005 A transient luminal chitinous matrix is required to model epithelial tube diameter in the Drosophila trachea. Dev. Cell 9: 423-430. https://doi.org/10.1016/j.devcel.2005. 07.012

Tsikala, G., D. Karagogeos, and M. Strigini, 2014 Btk-dependent epithelial cell rearrangements contribute to the invagination of nearby tubular structures in the posterior spiracles of Drosophila. Dev. Biol. 396: 42-56. https://doi.org/10.1016/j.ydbio. 2014.09.019

Turner, C. A., D. H. Mack, and M. M. Davis, 1994 Blimp-1, a novel zinc finger-containing protein that can drive the maturation of $\mathrm{B}$ lymphocytes into immunoglobulin-secreting cells. Cell 77: 297306. https://doi.org/10.1016/0092-8674(94)90321-2

Wilk, R., I. Weizman, and B. Z. Shilo, 1996 Trachealess encodes a bHLH-PAS protein that is an inducer of tracheal cell fates in Drosophila. Genes Dev. 10: 93-102. https://doi.org/10.1101/ gad.10.1.93

Wilk, R., B. H. Reed, U. Tepass, and H. D. Lipshitz, 2000 The hindsight gene is required for epithelial maintenance and differentiation of the tracheal system in Drosophila. Dev. Biol. 219: 183-196. https://doi.org/10.1006/dbio.2000.9619

Wu, V. M., J. Schulte, A. Hirschi, U. Tepass, and G. J. Beitel, 2004 Sinuous is a Drosophila claudin required for septate junction organization and epithelial tube size control. J. Cell Biol. 164: 313-323. https://doi.org/10.1083/jcb.200309134

Communicating editor: N. Perrimon 